\title{
Downregulation of high mobility group box 1 attenuates the severity of acute lung injury in endotoxemic mice
}

\author{
XIAOJUAN ZHANG, ZHENGGANG LUAN, YINGJIAN LIANG, YINA LIU and XIAOCHUN MA \\ Intensive Care Unit, First Affiliated Hospital of China Medical University, Shenyang, Liaoning 110001, P.R. China
}

Received April 7, 2014; Accepted January 7, 2015

DOI: $10.3892 / \mathrm{mmr} .2015 .3251$

\begin{abstract}
High mobility group box 1 (HMGB1) is a systemic inflammation-associated cytokine mediator. The aim of the present study was to examine the effect of the downregulation of HMGB1 in a lipopolysaccharide (LPS)-induced mouse model of acute lung injury (ALI). It was identified that serum levels of tumor necrosis factor- $\alpha$ and interleukin- $1 \beta$, lung myeloperoxidase activity and malondialdehyde content, as well as lung wet/dry weight ratios were all increased following LPS challenge. However, LPS-mediated increases in these parameters were significantly downregulated in HMGB1 small interfering (si)RNA-treated mice versus the negative control siRNA-treated mice. In addition, the administration of HMGB1 siRNA in LPS-treated mice resulted in a decreased DNA binding activity of nuclear factor $-\kappa \mathrm{B}(\mathrm{NF}-\kappa \mathrm{B})$ in the lung. It was demonstrated that downregulation of HMGB1 decreases inflammation and the severity of sepsis associated with ALI, possibly via inhibiting the NF- $\kappa$ B DNA-binding activity. The present data support HMGB1 as a contributor to the pathogenesis of LPS-induced sepsis and ALI.
\end{abstract}

\section{Introduction}

Sepsis is associated with a high mortality rate $(1,2)$. Acute lung injury (ALI) is characterized by an intense inflammatory process in the lung. It is the most common complication of severe sepsis as it is associated with the high rates of morbidity and mortality (3-7). Despite significant advances in understanding the pathogenesis and management of ALI in sepsis, the mortality rate remains high.

Lipopolysaccharide (LPS), the major endotoxin of gram-negative bacteria, may enter the bloodstream and elicit an acute systemic inflammatory response, which leads to sepsis and shock (8). Pro-inflammatory cytokines are involved in the

Correspondence to: Dr Xiaochun Ma, Intensive Care Unit, First Affiliated Hospital of China Medical University, 92 Bei-Er Road, Shenyang, Liaoning 110001, P.R. China

E-mail: xjzhang09@sina.com

Key words: acute lung injury, high mobility group box 1 , inflammation, small interfering RNA, nuclear factor- $\kappa \mathrm{B}$ development of tissue damage, metabolic acidosis, hypotension, multiple organ failure and even fatality during sepsis $(9,10)$. High mobility group box 1 (HMGB1) is a cytokine mediator that has been observed to have an important role in delayed endotoxin lethality and systemic inflammation in murine sepsis models (11-14). HMGB1 is highly expressed in the nucleus of endothelial cells, which are a crucial source of HMGB1 in inflammation $(15,16)$. As a pro-inflammatory cytokine, HMGB1 has been hypothesized to be an ideal target in sepsis therapy (17-21). There is clear evidence that anti-HMGB1 antibodies may protect against lethality, even if the first dose of antibody is administered $24 \mathrm{~h}$ after infection (22). A previous study has also identified chemically synthesized antisense oligonucleotides (23), which decrease HMGB1 expression and significantly inhibit inflammation in human umbilical vein endothelial cells (HUVECs). Therefore, gene transfer has been proposed as a novel method to produce cytokine inhibitors or antagonists to treat inflammation. However, the gene-silencing effects of HMGB1 via RNA interference (RNAi) in the development of ALI in sepsis remain to be elucidated. The aim of the present study was to determine whether the downregulation of HMGB1 reduced endotoxin-induced inflammation in mice.

\section{Materials and methods}

Animals. A total of 20 male C57BL/6 mice (age, 8-10 weeks) were obtained from the Experimental Animal Center of China Medical University (Shenyang, China). The mice were housed in rooms maintained at $20 \pm 2^{\circ} \mathrm{C}$ in a $12 \mathrm{~h}$ light/dark cycle for at least 1 week to acclimate to their surroundings, during which mice had access to water and standard mouse chow ad libitum. Mice were fasted overnight prior to experiments, but had access to water ad libitum. The animal study protocol was approved by the Institutional Animal Care and Use Committee of China Medical University.

HMGB1 small interfering RNA (siRNA). siRNA sequences targeting the coding regions of human HMGB1 were generated using the siRNA design center from GenScript Co., Ltd. (Nanjing, China). The siRNA sequences were inserted into the pRNA-U6.1/Neo vector attained from GenScript. Recombinant siRNA plasmids were transfected into HUVECs and the expression of HMGB1 was verified using western blot analysis (23). In brief, in order to construct pRNAU6.1/Neo-HMGB1, the human HMGB1 siRNA 
corresponding to the coding region (5'-AAGGTTGAGA GCTATTGCTGA-3') was synthesized using GenScript's siRNA design center (http://www.genscript.com/rnai.html) and inserted into pRNAU6.1/Neo using BamH1 and HindIII restricted enzymes. The recombinant pRNAU6.1/Neo-HMGB1 was then transfected into DH5 $\alpha$ cells. Additionally, the pRNA-U6.1/Neo vector was used as a control.

Animal grouping and treatment. Mice were randomly assigned to one of four groups $(n=5)$ : Control, LPS, LPS plus pRNA-U6.1/Neo-vector and LPS plus pRNA-U6.1/Neo-HMGB1. The pRNA-U6.1/Neo dosage was considered to be the optimum quantity based on a previous study (24). pRNA-U6.1/Neo-HMGB1 and pRNA-U6.1/Neo-vector $\left(3.6 \times 10^{8}\right.$ plaque forming units $/ 100 \mathrm{~g}$ in $25 \mu \mathrm{l}$ sterile phosphate-buffered saline solution; SD1201; GenScript Co., Ltd) was delivered via intravenous injection through the penile vein $48 \mathrm{~h}$ prior to LPS injection. A single dose of LPS (30 mg/kg body weight in $100 \mu 1$ saline; Escherichia coli serotype 055:B5; Sigma-Aldrich, St. Louis, MO, USA) was administered intraperitoneally.

Sample collection. Following LPS administration for $12 \mathrm{~h}$, mice were anesthetized via intraperitoneal injection with $0.1 \mathrm{ml} / 10 \mathrm{~g} 10 \%$ chloral hydrate and then euthanized through cervical dislocation. Serum and lung tissue samples were then collected. The superior lobe of the right lung was excised for histopathological examination. The middle lobe was excised for analysis of the lung wet/dry (W/D) weight ratio. The lower lobe was frozen in liquid nitrogen for a nuclear factor- $\kappa \mathrm{B}$ (NF- $\mathrm{kB})$ DNA-binding activity assay.

Lung W/D weight ratio. The middle lobe of each lung was blotted dry with filter paper for surface drainage and then weighed to obtain the wet weight. The lobe was then dried in a drying oven at $65^{\circ} \mathrm{C}$ for $72 \mathrm{~h}$; subsequently, the lung tissue was weighed to obtain the dry weight. The W/D ratio was calculated to assess tissue edema.

Enzyme-linked immunosorbent assay (ELISA). The levels of tumor necrosis factor- $\alpha$ (TNF- $\alpha$ ) and interleukin-1 $\beta$ (IL-1 $\beta$ ) in mouse blood samples were measured with commercially available ELISA kits (R\&D Systems, Minneapolis, MN, USA) according to the manufacturer's instructions. The sample levels were calculated by comparison to a standard curve and expressed as $\mathrm{pg} / \mathrm{ml}$.

Lung histopathology. Lung tissues were fixed in $10 \%(\mathrm{w} / \mathrm{v})$ neutral-buffered formalin for $24 \mathrm{~h}$, dehydrated in a graded ethanol series and subsequently embedded in paraffin. Sequential $5 \mu \mathrm{m}$ sections were stained with routine hematoxylin and eosin (H\&E) for morphological analysis. The slides were investigated under a light microscope (BA400 Binocular Microscope; Motic, Xiamen, China).

Measurements of myeloperoxidase (MPO) activity and malondialdehyde (MDA) in lung tissue. Frozen lung tissues were thawed, homogenized in cold saline at a ratio of 1:19 (weight/volume) and then centrifuged at $800 \mathrm{xg}$ for $10 \mathrm{~min}$ at $4^{\circ} \mathrm{C}$. The tissue supernatants were then collected for biochemical analyses. MPO activity and MDA levels in the lung tissue were determined using commercially available kits (Jiancheng Bioengineering Institute, Nanjing, China). For the MPO activity assays, the supernatants were incubated with hydrogen peroxide in the presence of $0.167 \mathrm{mg} / \mathrm{ml} \mathrm{O}$-dianisidine dihydrochloride for $30 \mathrm{~min}$. The change in absorbance at $460 \mathrm{~nm}$ for each sample was recorded with a plate reader (BioTek Instruments, Inc., Winooski, VT, USA). The MPO activity was defined as the quantity of enzyme degrading $1 \mu \mathrm{mol}$ peroxide $/ \mathrm{min}$ at $37^{\circ} \mathrm{C}$ and is expressed as units per gram lung tissue. The MDA content was determined based on the reaction of MDA with thiobarbituric acid (Sigma-Aldrich) at $90-100^{\circ} \mathrm{C}$. The MDA levels are expressed as nmol $/ \mathrm{mg}$ protein.

Electrophoretic mobility shift assay (EMSA). NF- $\mathrm{BB}$ DNA binding activity was measured with EMSA using nuclear extracts from lung tissue. Nuclear proteins were extracted using nuclear and cytoplasmic extraction reagent kits (Pierce Biotechnology, Rockford, IL, USA). End labeling of double-strand oligonucleotides containing the NF- $\kappa \mathrm{B}$ consensus sequence (5'-AGTTGAGGGGACTTTCCCAGGC-3') was performed using $\gamma^{-32} \mathrm{P}-\mathrm{dATP}$ (Amersham Pharmacia Biotech, Piscataway, NJ, USA) and T4-polynucleotide kinase (Takara Bio Inc., Ohtsu, Japan) at $37^{\circ} \mathrm{C}$ for $60 \mathrm{~min}$. Nuclear extracts $(20 \mu \mathrm{g})$ were incubated in a $20 \mu 1$ reaction mixture of $4 \%$ glycerol, $1 \mathrm{mM} \mathrm{MgCl}_{2}, 0.5 \mathrm{mM}$ EDTA, $0.5 \mathrm{mM}$ DTT, $50 \mathrm{mM} \mathrm{NaCl}, 10 \mathrm{mM}$ Tris- $\mathrm{HCl}$ (pH 7.5) and $50 \mu \mathrm{g} / \mathrm{ml}$ poly(deoxyinosinic-deoxycytidylic) acid (P4929; Sigma-Aldrich), with or without unlabeled oligonucleotide for $10 \mathrm{~min}$ at room temperature. ${ }^{32} \mathrm{P}$-labeled oligonucleotide probes were then added and incubated at room temperature for $20 \mathrm{~min}$. Electrophoresis of samples was performed on a 5\% polyacrylamide gel and visualized directly by autoradiography following drying the gel. Quantification was performed by image analysis using densitometry (ChemiDoc system; Bio-Rad, Hercules, CA, USA).

Statistical analysis. The results are expressed as the mean \pm standard deviation of at least three separate experiments. A one-way analysis of variance with Dunnett's multiple comparison tests was performed using SPSS software, version 10.0 (SPSS, Inc., Chicago, IL, USA). $\mathrm{P}<0.05$ was considered to indicate a statistically significant difference.

\section{Results}

Downregulation of HMGBI attenuates LPS-induced histopathological changes in lung tissue. To evaluate the histopathological characteristics of lung tissue in LPS-challenged mice, lung tissue sections were stained with H\&E. As shown in Fig. 1A, lung tissue from the sham group exhibited a normal structure and no histopathological changes. In the LPS-treated mice with or without pRNA-U6.1/Neo-vector injection, the lungs had widespread increased alveolar wall thickness caused by edema, severe hemorrhage in the alveolus, alveolus collapse and marked inflammatory cell infiltration (Fig. 1B and C). However, in the HMGB1 siRNA and LPS-treated groups, histopathological changes in the lung were attenuated when compared with those in the LPS plus pRNAU6.1/Neo-vector group, particularly the inflammatory cell infiltration findings (Fig. 1D). These results suggest that downregulation of HMGB1 may protect mice against LPS-induced lung injury. 


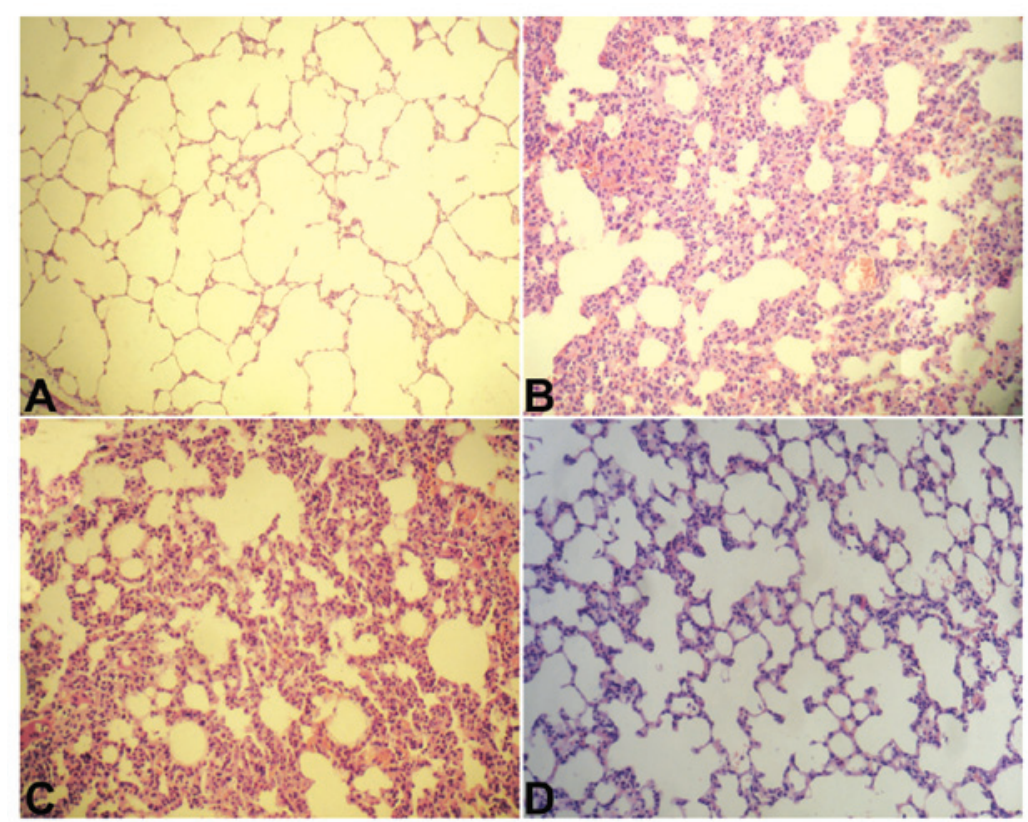

Figure 1. Histological examination of lung tissue stained with hematoxylin and eosin. (A) No evident pathological changes were observed in control mice. In (B) LPS-treated and (C) LPS plus pRNA-U6.1/Neo-vector-treated mice, lungs had widespread increased alveolar wall thickness caused by edema, severe alveolar congestion, alveolus collapse and marked inflammatory cell infiltration. (D) In LPS plus pRNA-U6.1/Neo-HMGB1-treated mice, the histological changes involved markedly reduced interstitial edema and inflammatory cell infiltration. Magnification, x200. Figure is representative of at least three experiments performed on different experimental days. LPS, lipopolysaccharide.
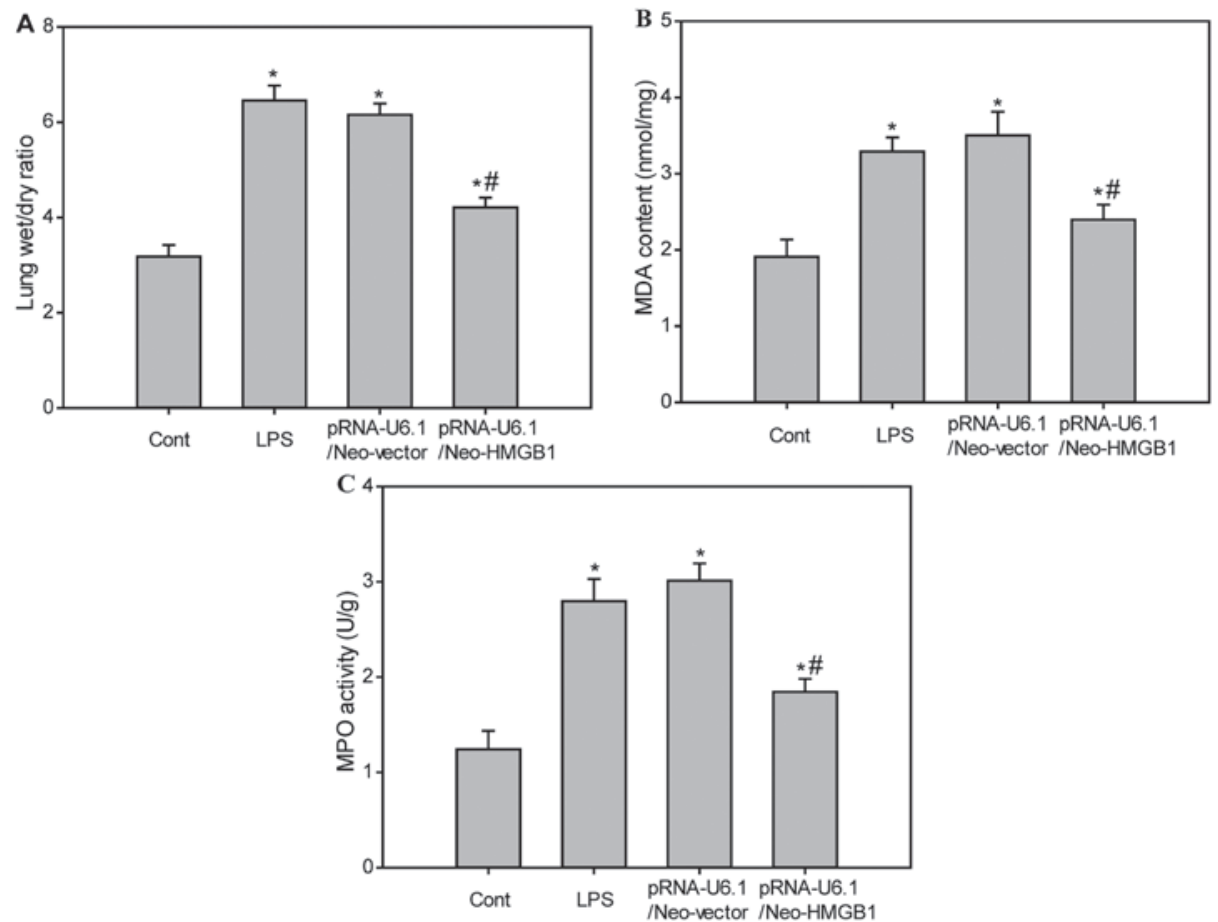

Figure 2. Effect of HMGB1 downregulation on the lungs. The effect of HMGB1 downregulation on (A) lung wet/dry ratio, (B) MDA content and (C) MPO activity in lung tissue of LPS-induced septic mice was observed. Mice were administered with pRNA-U6.1/Neo-HMGB1 following LPS administration. The lung wet/dry ratio, MDA content and MPO activity were determined at $12 \mathrm{~h}$ after LPS challenge. Values presented are the mean \pm standard deviation ( $\mathrm{n}=5$ ).

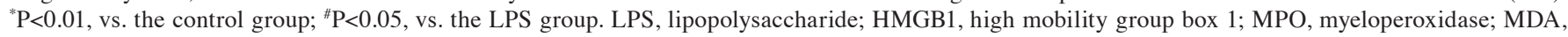
malondialdehyde.

Downregulation of $H M G B 1$ reduces lung W/D ratio, $M D A$ level and MPO activity in lung tissue of LPS-treated mice. The W/D ratio was used to evaluate pulmonary vascular permeability. When compared with the sham group, the lung
W/D ratios were significantly increased in the LPS group and LPS plus pRNA-U6.1/Neo-vector-treated group. No difference was identified between the LPS and LPS plus pRNA-U6.1/Neo-vector-treated groups. However, the increased 

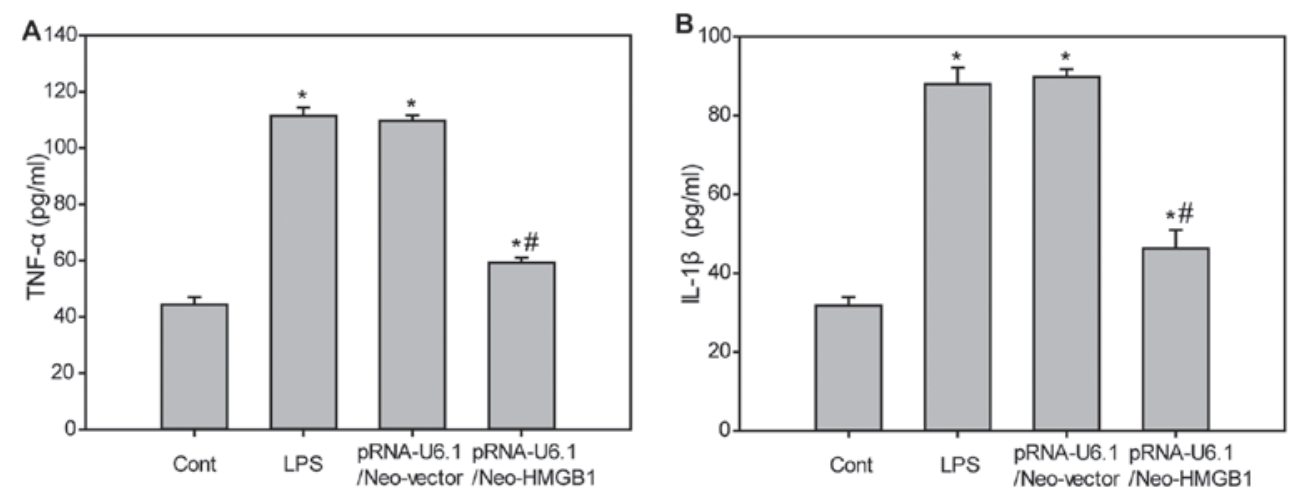

Figure 3. Effect of HMGB1 downregulation on the lungs. The effect of HMGB1 downregulation on the serum concentration of (A) TNF- $\alpha$ and (B) IL-1 $\beta$ of LPS-induced septic mice. Data are presented as the mean \pm standard deviation $(\mathrm{n}=5)$. ${ }^{\mathrm{P}}<0.01$, vs. the sham group; ${ }^{*} \mathrm{P}<0.01$, vs. the LPS group. TNF- $\alpha$, tumor necrosis factor- $\alpha$; IL-1 $\beta$, interleukin-1 $\beta$; LPS, lipopolysaccharide; HMGB1, high mobility group box 1.
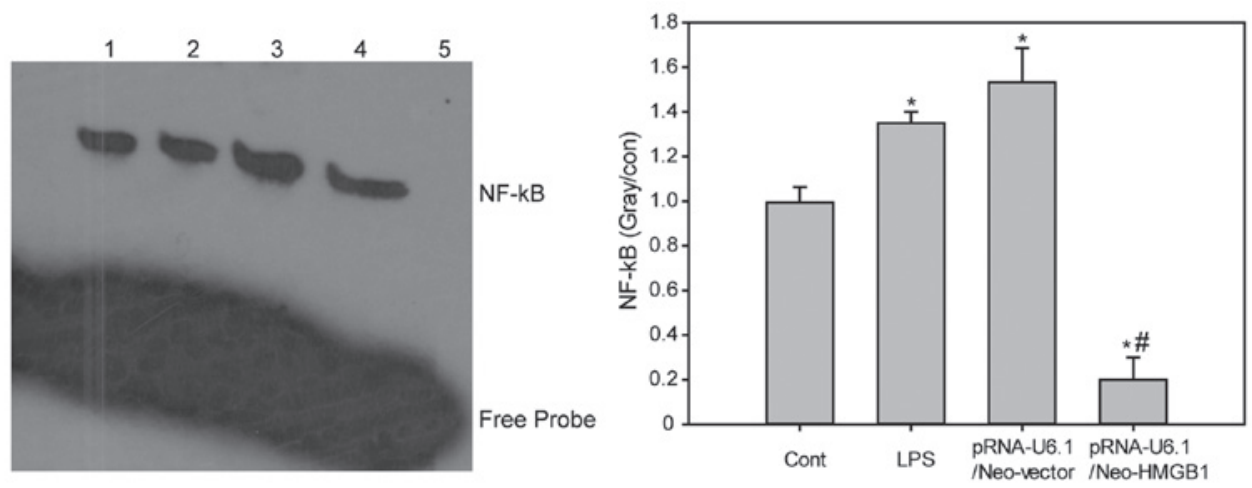

Figure 4. Pulmonary expression of NF- $\mathrm{BB}$ DNA binding activity in lung tissue from each group. NF- $\mathrm{BB}$ DNA binding activity in the lung was assessed at $6 \mathrm{~h}$ after sepsis in the control group (lane 1), sepsis group (lane 2), pRNA-U6.1/Neo-vector treated group (lane 3) and pRNA-U6.1/Neo-HMGB1 treated group (lane 4). Nuclear extracts were incubated with ${ }^{32} \mathrm{P}-$-labeled NF-kB consensus oligonucleotides and resolved by electrophoretic mobility shift assay. Unlabeled oligonucleotide (magnification, $\mathrm{x} 100$ ) was added to lane 5. Data shown are representative of three independent experiments. ${ }^{*} \mathrm{P}<0.05$, compared with the control group and ${ }^{\#} \mathrm{P}<0.05$, compared with the untreated sepsis group and pRNA-U6.1/Neo-vector treated group. NF- $\mathrm{kB}$, nuclear factor- $\mathrm{kB}$; LPS, lipopolysaccharide; HMGB1, high mobility group box 1.

lung W/D ratios in the LPS group were significantly reduced by treatment with HMGB1 siRNA (Fig. 2A; P<0.05), suggesting that downregulation of HMGB1 may prevent LPS-induced increases in lung vascular permeability. To assess neutrophil accumulation within pulmonary tissue, MPO activity was measured. Following LPS administration, MPO activity in lung tissue was significantly increased when compared with the control group. However, the increased MPO activity was markedly reduced following downregulation of HMGB1 (Fig. 2B; $\mathrm{P}<0.05)$. In addition, LPS administration significantly raised MDA levels in lung tissues and the increased MDA levels were suppressed by downregulating HMGB1 (Fig. 2C).

Downregulation of HMGBI inhibits production of inflammatory cytokines in LPS-challenged mice. Inflammatory cytokines, such as TNF- $\alpha$ and IL-1 $\beta$, are known to be involved in the pathophysiology of LPS-mediated pulmonary inflammation. Therefore, the serum levels of TNF- $\alpha$ and IL-1 $\beta$ were determined in LPS-challenged mice. As shown in Fig. 3, levels of serum TNF- $\alpha$ (Fig. 3A) and IL-1 $\beta$ (Fig. 3B) were minimally expressed in the control group. However, LPS administration markedly increased the expression of serum TNF- $\alpha$ and IL-1 $\beta$ levels, which were significantly attenuated following downregulation of HMGB1. These findings indicated that downregulation of HMGB1 inhibits LPS-induced production of inflammatory cytokines.

Downregulation of HMGB1 inhibits LPS-induced activation of $N F-\kappa B$. The NF- $\kappa$ B DNA binding activity in the lung tissue samples was determined. The NF- $\mathrm{B}$ DNA binding activity in the lung tissue of LPS-treated mice with or without negative control siRNA injection was increased when compared with that of the control group. By contrast, HMGB1 knockdown decreased LPS-induced increases in NF-kB DNA binding activity when compared with the LPS plus pRNAU6.1/Neo-vector group (Fig. 4; $\mathrm{P}<0.05$ ).

\section{Discussion}

In the present study, the effects of HMGB1 downregulation were investigated in LPS-induced ALI in mice. It was observed that HMGB1 siRNA-treated animals had a decreased lung W/D ratio compared with the negative control siRNA-treated mice. It is well-established that the overproduction of pro-inflammatory cytokines promotes the development of sepsis (25). Among these pro-inflammatory cytokines, TNF- $\alpha$ 
and IL-1 $\beta$, which are released within minutes after endotoxin exposure, are the most important early response cytokines. Previous studies have indicated that the persistent elevation of pro-inflammatory cytokines is associated with a worse outcome in patients with sepsis (26). In the present study, it was demonstrated that the serum concentrations of TNF- $\alpha$ and IL-1 $\beta$ increased significantly following LPS administration. However, these changes were markedly inhibited by HMGB1 siRNA treatment. In addition, using pRNA-U6.1/Neo-HMGB1 treatment significantly improved pulmonary histopathological findings and attenuated the severity of lung vascular permeability and edema.

$N F-\kappa B$ is a universal transcription factor that has a crucial role in regulating the transcription of over 200 genes, a number of which have important roles in the development of septic shock (27), including TNF- $\alpha$ and IL-1 (28). In the present study, it was identified that the downregulation of HMGB1 may inhibit the activation of NF- $\mathrm{NB}$ and consequently downregulate downstream inflammatory cytokines, relieve endothelial permeability and attenuate LPS-associated ALI in septic mice.

In conclusion, it was demonstrated that the downregulation of HMGB1 may inhibit LPS-induced inflammation and ALI, suggesting that it may provide a novel therapeutic strategy for sepsis.

\section{Acknowledgements}

The present study was supported by the National Natural Science Foundation of China (grant no. 81301619) and Shenyang Science and Technology plan projects (grant no. F13-220-9-11).

\section{References}

1. Angus DC, Linde-Zwirble WT, Lidicker J, Clermont G, Carcillo J and Pinsky MR: Epidemiology of severe sepsis in the United States: analysis of incidence, outcome, and associated costs of care. Crit Care Med 29: 1303-1310, 2001.

2. Fry DE: Sepsis, systemic inflammatory response, and multiple organ dysfunction: the mystery continues. Am Surg 78: 1-8, 2012

3. Ware LB and Matthay MA: The acute respiratory distress syndrome. N Engl J Med 342: 1334-1349, 2000.

4. Matthay MA and Zemans RL: The acute respiratory distress syndrome: pathogenesis and treatment. Annu Rev Pathol 6: 147-163, 2011.

5. Matthay MA and Zimmerman GA: Acute lung injury and the acute respiratory distress syndrome: four decades of inquiry into pathogenesis and rational management. Am J Respir Cell Mol Biol 33: 319-327, 2005.

6. Erickson SE, Martin GS, Davis JL, Matthay MA, Eisner MD and NIH NHLBI ARDS Network: Recent trends in acute lung injury mortality: 1996-2005. Crit Care Med 37: 1574-1579, 2009.

7. Zimmerman JJ, Akhtar SR, Caldwell E and Rubenfeld GD: Incidence and outcomes of pediatric acute lung injury. Pediatrics 124: 87-95, 2009.
8. Hudson LD, Milberg JA, Anardi D and Maunder RJ: Clinical risks for development of the acute respiratory distress syndrome. Am J Respir Crit Care Med 151: 293-301, 1995.

9. Gando S: Microvascular thrombosis and multiple organ dysfunction Syndrome. Crit Care Med 38 (Suppl 2): S35-S42, 2010.

10. Levi M and van der Poll T: Inflammation and coagulation. Crit Care Med 38 (Suppl 2): S26-S34, 2010.

11. Andersson U, Wang H, Palmblad K, et al: High mobility group 1 protein (HMG-1) stimulates proinflammatory cytokine synthesis in human monocytes. J Exp Med 192: 565-570, 2000.

12. Wang H, Bloom O, Zhang M, et al: HMG-1 as a late mediator of endotoxin lethality in mice. Science 285: 248-251, 1999.

13. Wang H, Yang H and Tracey KJ: Extracellular role of HMGB1 in inflammation and sepsis. J Intern Med 255: 320-331, 2004.

14. Fiuza C, Bustin M, Talwar S, et al: Inflammation-promoting activity of HMGB1 on human microvascular endothelial cells. Blood 101: 2652-2660, 2003.

15. Mullins GE, Sunden-Cullberg J, Johansson AS, et al: Activation of human umbilical vein endothelial cells leads to relocation and release of high-mobility group box chromosomal protein 1 . Scand J Immunol 60: 566-573, 2004.

16. Bae JS and Rezaie AR: Activated protein $\mathrm{C}$ inhibits high mobility group box 1 signaling in endothelial cells. Blood 118: 3952-3959, 2011

17. Yasuda T, Ueda T, Takeyama Y, et al: Significant increase of serum high-mobility group box chromosomal protein 1 levels in patients with severe acute pancreatitis. Pancreas 33: 359-363, 2006

18. Gibot S, Massin F, Cravoisy A, et al: High-mobility group box 1 protein plasma concentrations during septic shock. Intensive Care Med 33: 1347-1353, 2007.

19. Gaïni S, Pedersen SS, Koldkjaer OG, Pedersen C and Møller HJ: High mobility group box-1 protein in patients with suspected community-acquired infections and sepsis: a prospective study. Crit Care 11: R32, 2007.

20. van Zoelen MA, Laterre PF, van Veen SQ, et al: Systemic and local high mobility group box 1 concentrations during severe infection. Crit Care Med 35: 2799-2804, 2007.

21. Karlsson S, Pettilä V, Tenhunen J, Laru-Sompa R, Hynninen M and Ruokonen E: HMGB1 as a predictor of organ dysfunction and outcome in patients with severe sepsis. Intensive Care Med 34: 1046-1053, 2008.

22. Silva E, Arcaroli J, He Q, et al: HMGB1 and LPS induce distinct patterns of gene expression and activation in neutrophils from patients with sepsis-induced acute lung injury. Intensive Care Med 33: 1829-1839, 2007.

23. Zhang XJ, Luan ZG and Ma XC: shRNAs targeting high-mobility group box-1 inhibit E-selectin expression via homeobox A9 in human umbilical vein endothelial cells. Mol Med Rep 7: $1251-1256,2013$

24. Luan ZG, Zhang XJ, Yin XH, et al: Downregulation of HMGB1 protects against the development of acute lung injury after severe acute pancreatitis. Immunobiology 218 : 1261-1270, 2013.

25. Cannon JG, Tompkins RG, Gelfand JA, et al: Circulating interleukin-1 and tumor necrosis factor in septic shock and experimental endotoxin fever. J Infect Dis 161: 79-84, 1990.

26. Minamino T and Komuro I: Regeneration of the endothelium as a novel therapeutic strategy for acute lung injury. J Clin Invest 116: 2316-2319, 2006.

27. Pahl HL: Activators and target genes of Rel/NF-kappaB transcription factors. Oncogene 18: 6853-6866, 1999.

28. Rakonczay Z Jr, Hegyi P, Takács T, McCarroll J and Saluja AK: The role of NF-kappaB activation in the pathogenesis of acute pancreatitis. Gut 57: 259-267, 2008. 